\title{
Developing and Comparing Single-arm and Dual-arm Regrasp
}

\author{
Weiwei Wan and Kensuke Harada
}

\begin{abstract}
The goal of this paper is to develop efficient regrasp algorithms for single-arm and dual-arm regrasp and compares the performance of single-arm and dual-arm regrasp by running the two algorithms thousands of times. We focus on pick-and-place regrasp which reorients an object from one placement to another by using a sequence of pick-ups and placedowns. After analyzing the simulation results, we find dual-arm regrasp is not necessarily better than single-arm regrasp: Dualarm regrasp is flexible. When the two hands can grasp the object with good clearance, dual-arm regrasp is better and has higher successful rate than single-arm regrasp. However, dualarm regrasp suffers from geometric constraints caused by the two arms. When the grasps overlap, dual-arm regrasp is bad. Developers need to sample grasps with high density to reduce overlapping. This leads to exploded combinatorics in previous methods, but is possible with the algorithms presented in this paper. Following the results, practitioners may choose singlearm or dual-arm robots by considering the object shapes and grasps. Meanwhile, they can reduce overlapping and implement practical dual-arm regrasp by using the presented algorithms.
\end{abstract}

\section{INTRODUCTION}

Regrasp can be performed by a single robotic arm plus an extrinsic table surface[1]. The single arm changes the grasps taking advantage of the table. It picks up the object from its initial placement on the table, reorientates it to a new state and place it down on the table, and changes the grasps to pick it up again from the new state. In this way, a single arm can finish difficult reorientation tasks like flipping where the object cannot be directly placed down into the goal state.

Regrasp can also be performed by a dual-arm robot[2]. The robot changes the grasps by handling the object from one hand to another. One arm of the robot picks up the object from its initial placement on the table, reorientates it to a handling configuration in the work space and hold it. The other arm grasps the object from the handling configuration and reorientates it again. In this way, dual-arm robots can also finish difficult orientation tasks.

We wonder which has higher performance in pick-andplace regrasp. We develop efficient regrasp algorithms for both single-arm and dual-arm regrasp and compare their successful rates and time cost by running the algorithms thousands of times. We demonstrate the algorithms developed in this paper are fast enough for thousands of simulation and concluded that dual-arm regrasp is not necessarily better than single-arm regrasp. by analyzing the simulation results. The performance of dual-arm regrasp depends on object shapes and the overlapping of the grasps from the two hands. Practitioners may choose single-arm or dual-arm robots by

Weiwei Wan and Kensuke Harada are affiliated with the Manipulation Research Group, National Institute of Advanced Industrial Science and Technology (AIST). wan-weiwei@aist.go.jp considering the object shapes and grasps. Meanwhile, they can reduce overlapping and implement practical dual-arm regrasp by using the algorithms presented here.

While this paper concentrates on the algorithm development and the comparison of the single-arm and dual-arm regrasp, the results are also expected to be used to automatically decide arm numbers: Robots can use the algorithms to to predict whether she should use a single arm or dual arms.

\section{Related Work}

\section{A. Regrasp}

Most of the early work on regrasp planning uses a single arm. The seminal study is done by Tournassound et al.[3], and is later described in detail in the Handey system[4]. This early study builds a Grasp-Placement (GP) table to search for regrasp sequences. It solves the IK and checks all collisions to invalidate the grasp and placement pairs, fill up the GP table, and search the table to find a sequence of pick-andplace motion. There are lots of work following this study. For example, Rohrdanz et al.[5] improves the efficiency of regrasp planning using an evaluated breadth-first search and rated grasp and placement qualities. Terasaki et al.[6] adds a simple rotating mechanism to the robotic gripper, making the regrasp planning dynamic. Their study not only regrasps objects using pick-and-place, but also pivoting [7]. Stoeter et al.[8] replaces the GP table with a space of compatible grasp-placement-grasp triplets and search this space to find a sequence of pick-and-place motion. More practically, Cho et al.[9] implements the regrasp algorithm with a real robot using off-line mapping and on-line retrieving. During the offline mapping, they sample the workspace and pre-build a look-up table to hold the IK-feasible grasps at the sampled positions. During the on-line retrieving, they check the lookup table to quickly know whether regrasp is feasible. Using the look-up table avoids explicit IK solving and improves efficiency.

More recent work studies regrasp in the context of hierarchical TAsk and Motion Planning (TAMP) and solves the constraint satisfaction problem. The framework is presented by Lozano-Perez et al.[10] where a symbolic planner plans a sequence of high-level operations and a Constraint Satisfaction Problem (CSP) solver solves low-level problems. Under this framework, regrasp planning is divided into the symbolic pick-and-place sequence and the geometrically feasible placements, paths, grasps, and locations. A robot decides high-level operations using the symbolic planner and decides low-level operations using the CSP solver. LozanoPerez demonstrates the framework using a single-arm of a PR2 robot. The most challenging part of this framework is 
the exploded low-level combinatorics (see Dogar et al.[11]). Properly rating regrasps is essential to use this framework to solve problems with large constraint graphs. A similar framework is presented by Lagriffoul et al.[12]. Both singlearm and dual-arm regrasp are performed to demonstrate the framework.

In our previous work, we develop a single-arm regrasp algorithm using a novel graph and use the algorithm to analyze the utility of tilted work surfaces[1]. This paper continues the development by extending the algorithm to dual-arm robots. It uses the regrasp algorithms to compare the performance of single-arm and dual-arm regrasp.

\section{B. Dual-arm regrasp}

The difficulty of dual-arm regrasp is the high-dimensional configuration space composed by the two arms and the exploded number of combinatorics between the two grasps during handling. The seminal work discussing this difficulty is by Koga et al.[13]. The paper compares three 2D demos - two 2-DoF arms without obstacles, two 2-DoF arms with obstacles, and two 3-DoF arms with obstacles. The first demo can be exhaustively computed. The second demo employs manipulation graph et al.[14] to make computation feasible. The third one uses random sampling to further improve efficiency.

Following this initial study, lots of research are devoted to the dual-arm regrasp problem. Koga himself extends the $2 \mathrm{D}$ work to $3 \mathrm{D}$ regrasp planning using two and three manipulators and a small amount of manually specified grasp assignments[15]. The extension shows multi-robot regrasp is possible using the limited computational resources at that time. Saut et al.[2] studies the dual-arm regrasp problem by using an optimized regrasp position and object orientation. The optimized regrasp position minimizes wrist motions and the optimized object orientation minimizes the approaching angles of the two hands. The work is based on a roadmap composition work introduced by Gharbi et al.[16]. It fails to output a solution in some situations. Balaguer et al.[17] studies the dual-arm regrasp problem by estimating the two grasping positions for the two hands in the object?s local coordinate system. It optimizes the regrasp position and object orientation by minimizing the time needed to move the two hands to the estimated positions. Approaching directions of the hands are optimized later. Comparing with Saut, the algorithm runs at only one object orientation, but with more hand approaching directions. Vahrenkamp et al.[18] studies the dual-arm regrasp problem by pre-building both a singlearm manipulability map and a bi-manual manipulability map in work space. They query the single-arm data map to find the IK-feasible grasps of each hand and query the bi-manual data map to assign scores to the possible two-hand combinations. Their work is resolution-complete and can perform on-line search in 20ms. Most up-to-date, Suarez et al.[19] proposes employing the synergy analysis which was initially discussed and used in robotic grasping by Santello et al.[20] to reduce the computational cost of dual-arm regrasp. They compute a PMDs (Principle Motion Directions) manifold in the configuration space of the dual-arms, and choose the grasp configurations that have smaller distance to the PMDs manifold to reduce dual-arm combinatorics. Interestingly, this study has the advantage in getting a human-friendly robot motion.

Our dual-arm regrasp algorithm is based on the regrasp graph. It is somewhere between [2] and [18], and can be used on-line. In detail, we use manipulability and approachability to find an optimized position and use sampling to generate a sequence of orientation. Each position and orientation pair is a handling configuration, and the dual-arm robot performs regrasp at it. Our algorithm is complete and fast enough to find a motion sequence for difficult regrasp tasks like flipping. Most importantly, we compare single-arm and dualarm regrasp by running the algorithms thousands of times on random initial and goal states. To the best of our knowledge, this is the first work that compares the performance of singlearm and dual-arm regrasp.

\section{Overview of the Algorithms}

In this section, we present the algorithm flowcharts of single-arm and dual-arm regrasp and discuss briefly the role of each part in the flowcharts. The details will be discussed in later sections. (a) Single-arm Regrasp
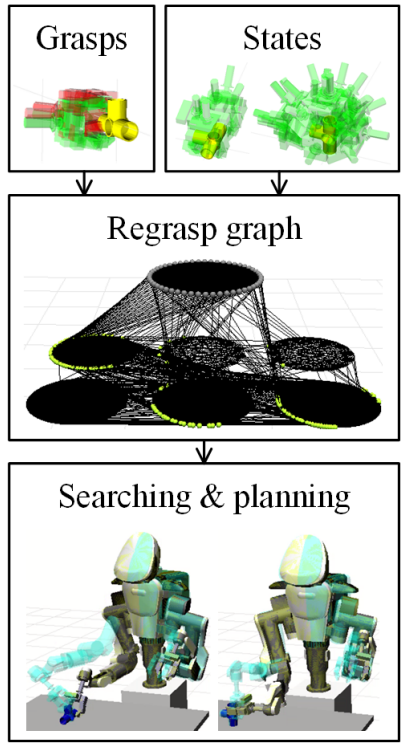

(b) Dual-arm Regrasp

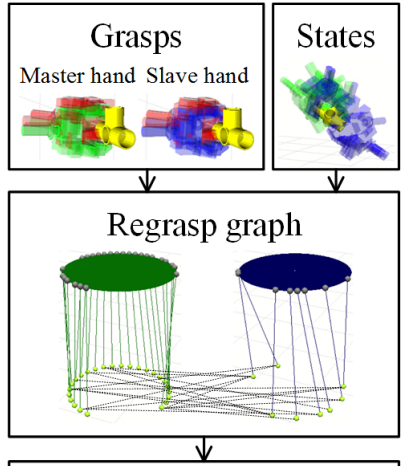

Searching \& planning

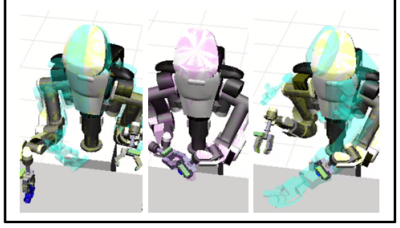

Fig. 1. Flow charts of the two regrasp algorithms developed for singlearm and dual-arm regrasp. In the single-arm case, we first plan the grasps and states (placements) using the object model (rendered in yellow) and the robot's hand model (rendered in red or green, depending whether the hand and the object collide with each other or not). Then, we build a regrasp graph using the states and their associated grasps. Third, we search the regrasp graph to find a regrasp sequence and do motion planning. The dual-arm case follows the same flow but involves two hands and a merged regrasp graph. The green hand and sub-graph denote the master and the blue hand and sub-graph denote the slave. The master hand handles the object to the slave hand. 


\section{A. Single-arm Regrasp}

The flowchart of single-arm regrasp is shown in Fig 11a). In the first step, we compute the grasps and states used for regrasp planning. The input of this step is the model of a robotic gripper and an object. The output includes (1) the resolution complete force-closure grasps of the robotic gripper on the object, and (2) the stable placements of the object on a table surface and the grasps associated with each placement. The component that computes the grasps is shown in the box named "Grasps". The component that computes the placements and its associated grasps is shown in the box named "States". In the second step, we build a regrasp graph using the grasps and states computed in step one. Each circle in the graph represents one placement of the object, and each node on the circle represents one grasp. The edges encode the relationship between the grasps and the placements: One can be changed into another by the robot using transfer or transit motions[21]. The last step is to search the regrasp graph and do motion planning. The graph search finds is a sequence of pick-and-place operations and is in the high level of task planning. The motion planning finds a sequence of arm trajectories that connects adjacent pick-and-place operations in the high-level sequence.

\section{B. Dual-arm Regrasp}

The flowchart of dual-arm regrasp is shown in Fig 1 b). Like the single-arm regrasp, the dual-arm regrasp also includes a grasp-and-state planning step, a regrasp graph building step, and a searching and motion planning step. The difference is: First, both hands are considered in the first step. The grasp planning computes the collision-free grasps of both hands. The state planning component associates the available grasps of both hands to each placement. Second, the state planning not only compute the placements on a table, but also the handling configurations in the air. The handling configurations are the positions and orientations of the object for dual-arm handling. The state planning uses the approachability and manipulability to find an optimized position and uses sampling to generate a sequence of rotation. Each position and rotation pair is a candidate handling configuration. The state planning associates the grasps of both hands to the handling configurations. Collision detections between hands are performed during the association. Third, the regrasp graph is composed of two sub-graphs, one for the master hand and one for the slave hand. The circles in the higherlayer of each sub-graph are the initial and goal placements of the object on the table. The circles in the lower-layer of each sub-graph are the handling configurations. The two sub-graphs are connected at the lower-layer. It is where the master hand handles the object to the slave hand.

\section{Grasps and States}

\section{A. Grasp planning}

In detail, our grasp planning is done in the local coordinate system of the object. The flow is shown in Fig 2 .

First, we cluster the mesh model of the object and sample contact points. The clustering will merge coplanar triangles

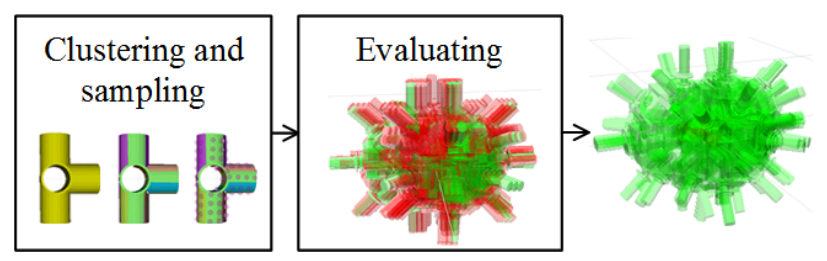

Fig. 2. Grasp planning in the local coordiante system of the object. In the left, the object surface is clustered and sampled. In the middle, each pair of the sampled points from two parallel clusters is evaluated using grasp stability, force-closure, and collision. In the right, low-quality grasps are removed (denoted in red in the middle figure).

on the mesh model using a tolerance value given by the user. On each cluster, we sample contact points using its two principal axes. Suppose the bounding box of the cluster is $\left\{\left(e_{1}^{\min }, e_{1}{ }^{\max }\right),\left(e_{2}^{\min }, e_{2}{ }^{\max }\right)\right\}$ and the two principal axes are $\left\{x_{1}, x_{2}\right\}$, we sample the surface following $p=\omega_{1} x_{1}+\omega_{2} x_{2}$, $\omega_{1} \in\left[e_{1}{ }^{\min }, e_{1}{ }^{\max }\right], \omega_{2} \in\left[e_{2}{ }^{\min }, e_{2}{ }^{\max }\right]$. The step is shown in the box named "Clustering and sampling" in Fig 2 . Then, we evaluate each pair of contact pairs from two parallel clusters by checking the grasp stability, force-closure, and collision. For grasp stability, we remove the contact points that has a small offset to cluster boundary and low resistance to external torques. For force-closure, we measure each two contact points on parallel clusters with eight approaching directions, check if it has large wrench space ball, and remove the grasps that have low quality. For collision, we remove the grasps that induce collision between fingers and collision with the object. This step is shown in the box named "Evaluation" in Fig 2 The removed grasps are drawn in red in the figure. In contrast, the remaining grasps are drawn in green.

The output of grasp planning is the grasps in the object's local coordinate system. Only the grasps of one hand is computed in single-arm regrasp. The grasps of both hands are computed in dual-arm regrasp.

\section{B. State planning}

There are two kinds of states. One is the stable placements of the object on a table surface, used in both single-arm and dual-arm regrasp. The other one is the handling configurations of the object in the air, used only in dual-arm regrasp. The flow charts of computing these two kinds of states are shown in Fig 3 (a) and (b) respectively.

1) Placement planning: The placement planning includes three steps. In the first step, we compute the convex hull of the original object mesh and perform surface clustering on the convex hull. The surface clustering algorithm is the same as the one used in grasp planning. Each cluster is one candidate standing surface and the object may be placed on it. Then, we test the stability of the objects standing on these candidate surfaces. This is a filtering process and the unstable placements, e.g. the placements where the Zero Moment Point (ZMP) is outside the candidate surface or too near to its boundary, will be removed. In the third step, we associate the grasps to the stable placements filtered by step two: (1) We remove the grasps that collide with the table surface and 
(a) Single-arm Regrasp

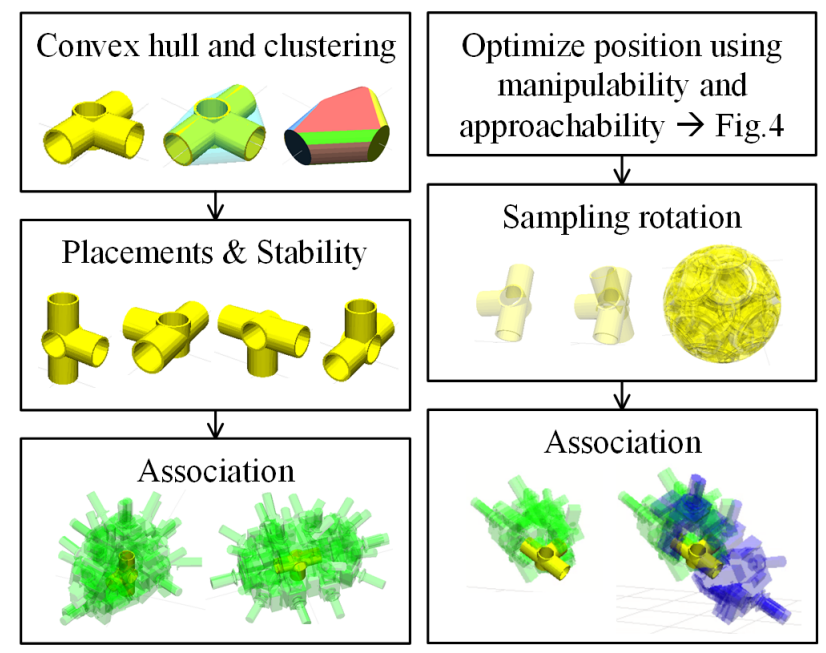

Fig. 3. Computing the stable placements for single-arm regrasp and both the stable placements and the handling configurations for dual-arm regrasp. In the single-arm case, we cluster the convex hull of the object (top box) and test if any of the clusters could be the standing surface of a stable placement (middle box). Each stable placement is associated with available grasps (rendered in green in bottom box). In the dual-arm case, we fix the object to an optimized position and sample the orientation (middle box). Each position and rotation pair is a candidate handling configuration and is associated with the available grasps of both hands (rendered in red and blue in bottom box).

(2) we transform from the object?s local coordinate system to each placement?s local coordinate system.

The placements and their associated grasps are described in local coordinate systems. We perform collision detection to ensure the hand, the object, and the table surface do not collide with each other. However, the yaw of the object on the table surface and the IK of the robot arm are not computed. They are delayed to regrasp graph searching.

2) Handling configuration planning: We use an heuristic method to plan the handling configurations for handling. First, an optimized position is computed using manipulability and approachability. This step involves three levels of discretization. In level 1, we sample the surface in the middle of the two arms with grids. In level 2, at each of the sampled grid, we sample the approaching directions pointing at it. In level 3, we sample the rotation around each sampled approaching direction. After the three levels of discretization, we get a list of rotation matrices representing the configurations of the hand to grasp something on the middle surface. We move the hand to these configurations and compute the manipulability of the arm using $\sqrt{\operatorname{det}\left(\mathbf{J J}^{T}\right)}$ [22]. There are two possible cases. One is IK-infeasible configuration. The robot cannot approach the grid using the rotation matrix in this case and the manipulability is 0 . The other is the IK feasible configuration where the manipulability has a positive value. If the manipulability of one rotation matrix is not 0 and is larger than a threshold value, we count it as approachable. At each sampled grid, we count the number of approachable rotation matrices and use the value as the approachability. The manipulability and approachability are computed following these routines and are used to optimize the position of handling. The rotation matrices, the manipulability, and the approachability are shown in Fig 4 The left part of Fig 4 shows the rotation matrices. The purple vectors are the approaching directions and the rotation matrices are sampled around the approaching directions. The right part of Fig 4 shows the manipulability and approachability. The manipulability at the grid along different approaching directions is rendered with a color spectrum in the the-lighter-the-higher style. The approachability is rendered by the number of vectors at each grid.
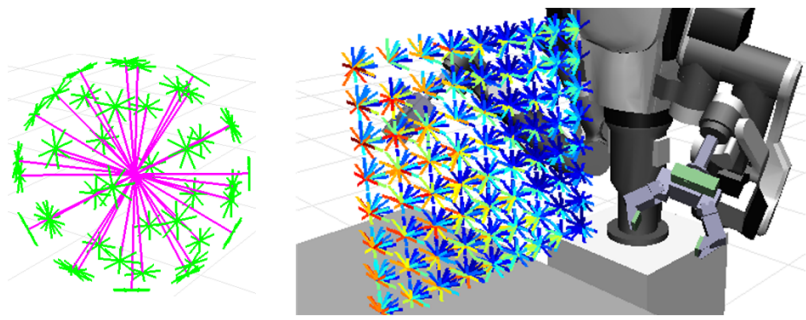

Fig. 4. Optimizing the handling position using manipulability and approachability. First, we discretized the middle surface in front of the robot with lattice (see right figure). Then, for each grid, we sample the approaching directions pointing at it (see the purple vectors in left figure). Third, we sample the rotation around each approaching direction (see the green vectors at the end of each purple vector). A list of configurations is generated through the discretization. We compute the manipulability at each configuration, count the number of rotation with good manipulability at each grid, and save the value as the approachability. The grid with highest approachability is used as the optimal handling position.

We choose the grid position that has largest approachability as the optimized position for handling. This is because at this position the two hands can approach an object with a large number of directions as well as high manipulability. The optimized position will be the translational component of the handling configuration.

The rotational component of the handling configuration is evenly sampled. We compare different heuristic strategies for the rotational component and conclude that even sampling is the most effective one. The second box in Fig 3 (b) shows the evenly sampled rotations. The left figure shows the object at the first rotation matrix. The middle one shows both the object at the first and second rotation matrices. The right figure shows the overlap of all rotation.

Each position and rotation pair is a candidate handling configuration and we associate it with the grasps of both hands. This is done on-line by checking the collisions between the hands and their approaching directions (the hand directions that induce crossing arms are also removed in this step). Meanwhile, we rate the quality of each handling configuration using its angular distance to the goal. The handling configuration that has a shorter distance to the goal is set with higher priority and will be tried first. More exactly, we loop the handling configurations using the second level of discretization during the handling trial. That is, the most outside loop is the approaching vectors. We try the nearest handling configuration to the goal at each approaching vector in the first loop, try the second-nearest handling configuration 
in the second loop, and so on. The looping process will be further discussed in Section V.B.

\section{Building and Searching the Regrasp Graphs}

Fig 5 shows the flow charts of building the regrasp graphs. The grasps associated with the same state are connected using transit edges and the grasps associated with different states are connected using transfer edges. The difference between single-arm regrasp graph and dual-arm regrasp graph is: In single-arm case, the regrasp graph is built once and dynamically trimmed using the results of searching. In dual-arm case, the regrasp graph is built dynamically using the selected handling configuration. (a) Single-arm Regrasp

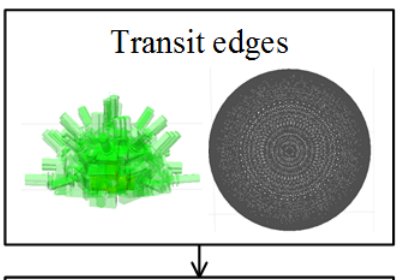

Transfer edges
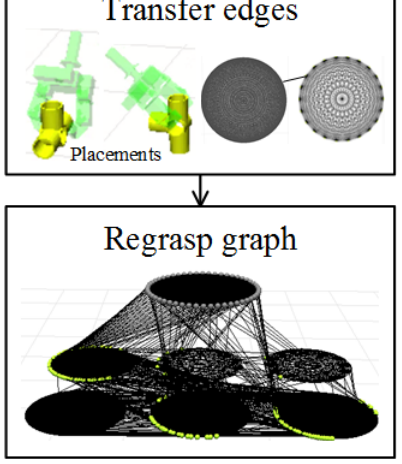

(b) Dual-arm Regrasp
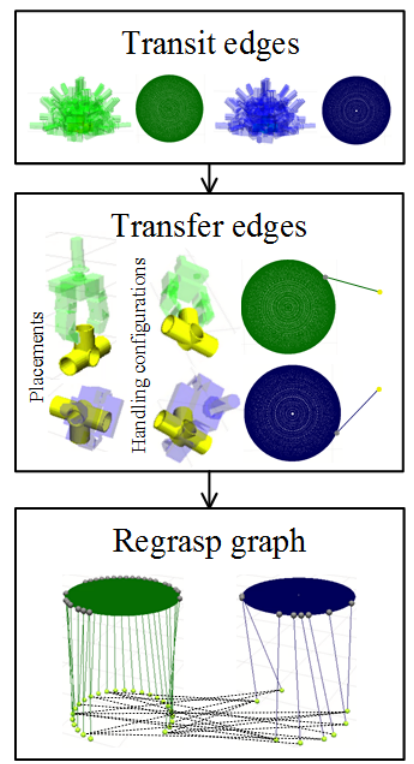

Fig. 5. Building the regrasp graphs. In the single-arm case, we first arrange the grasps associated with the same placement around a circle, and make them fully connected (top box). Then, we connect two circles by checking if they share a common grasp (e.g. the same grasp described in the object's local coordinate system). Using these two steps, we get a graph like the one shown in the bottom box. The dual-arm case involves both placements and handling configurations. The placements are similar to the single-arm case and encode transit motion using fully connected circles (see the green and blue circles in top and middle boxes). The grasps associated with handling configurations are also arranged around circles, but they are not connected inside the circle. The lower layer in the bottom box shows this kind of circles.

\section{A. Single-arm case}

In single-arm case, we only build the regrasp graph for one time. First, we arrange the grasps associated with the same state around a circle and make them fully connected. The edges inside the circle are transit type since the grasp at one end of the edge can be changed to the grasp at the other end without moving the object. The figures in the top box of Fig 5 a) illustrates one of the arrangement and connection. The circle on the right corresponds to the grasps and placement on the left. Then, we connect the circles. If two grasps associated with different states are the same in the object?s local coordinate system, we add an edge between them. The edges connecting the circles are transfer type since the grasp at one end of the edge can be changed to the grasp at the other end by moving the object. Finally, we get a regrasp graph like the figure in the bottom box. Each circle represents one state, and each node on the circle represents one grasp. Edges inside the circle represents transit motion and edges connecting the circles represent transfer motion. The grasp has two layers where the upper one only includes the initial placement and the lower layer has all possible placements. This two-layer structure enables both simple pick-and-place planning, namely pick-and-place with the same placement, and reorientating planning that picks and places at different placements.

Given the initial placement and goal placement, we search the regrasp graph to find a sequence of pick-and-place operations. The starting node of searching is a random grasp on the circle in the upper layer and the ending node is a random grasp on the circle that corresponds to the end placement in the lower layer. The result of searching is a sequence of states, grasps, and transit and transfer motion connecting the starting and ending nodes. For each state and grasp in the searched result, we check if they are IKfeasible and collision-free. In the placement planning part, we only check the collisions between the hand, the object, and the table. The IK solving and the collision detection between the robot and other obstacles in the environment are delayed to this searching step. If the sequence is IKinfeasible or collides with obstacles, we trim the regrasp graph by removing the correspondent grasps and search again. Or else, we do motion planning using Transition-based RRT[23] for the edges in the searched result. Like the grasps, we trim the regrasp graph by removing the correspondent edges and search again if motion planning fails. Fig 6 shows this process. Either an available sequence is found or the regrasp graph becomes unconnected after the search.

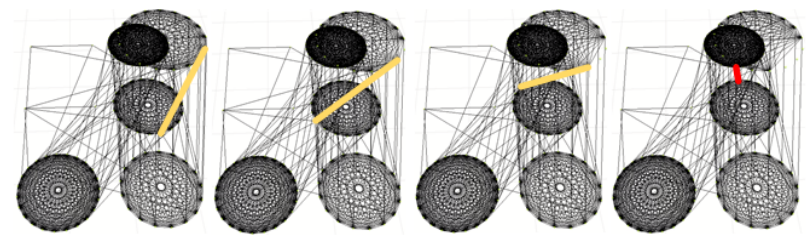

Fig. 6. Searching the single-arm regrasp graph. The single-arm regrasp is built once but the edges and nodes are dynamically trimmed during searching and motion planning. The first three paths (rendered in yellow) include unavailable nodes or edges. They are deleted and searched again.

\section{B. Dual-arm case}

In dual-arm case, we loop the rated handling configurations and rebuild the regrasp graph at each loop. The regrasp graph is composed of two sub-graphs. One for the master hand and one for slave hand. Each sub-graph is composed of only two states: One is the initial or goal placement, the other is the handling configuration. The green and blue plots in the bottom box of Fig 5.b) show the two sub-graphs. The upper layer of the green plot is the initial placement and the upper layer of the blue plot is the goal placement. They are built in 
the same way as the placement circles in the single-arm case. Instead of delaying IK-solving and collision detection to the searching step, we do them before starting the loop: Only the available grasps are used to build regrasp graphs. The lower layers of the plots are the handling configurations. The two sub-graphs share a same handling configuration and are bridged at it. The edges connecting the upper layer and the lower layer indicate the transfer motion. If a grasp associated with the handling configuration and a grasp associated with the initial or goal placement are the same in the object?s coordinate system, we connect them with a transfer edge.

At the handling configuration, the robot cannot perform transit motion with a single arm and there is no edges inside the circle. But the robot can transit bi-manually: It could hold the object with one hand and perform transit motion with the other hand. Therefore, we connect the two circles at the lower layers of the sub-graphs using transit edges, which are illustrated with dotted lines in Fig 5. The nodes on the lower circles and the edges connecting them change as we select different configurations and rebuild the regrasp graphs. IK-solving and collision detection at the nodes on the lower circles are performed each time the regrasp graph is rebuilt. During searching, we no long need to waste time on IK. However, further checking the collision between master and slave arms is required. The collision detection done during rebuilding the graph was performed independently on two arms. The collision between master and slave arms need to be further checked before merging the two sub-graphs. Fig 7 shows the process.

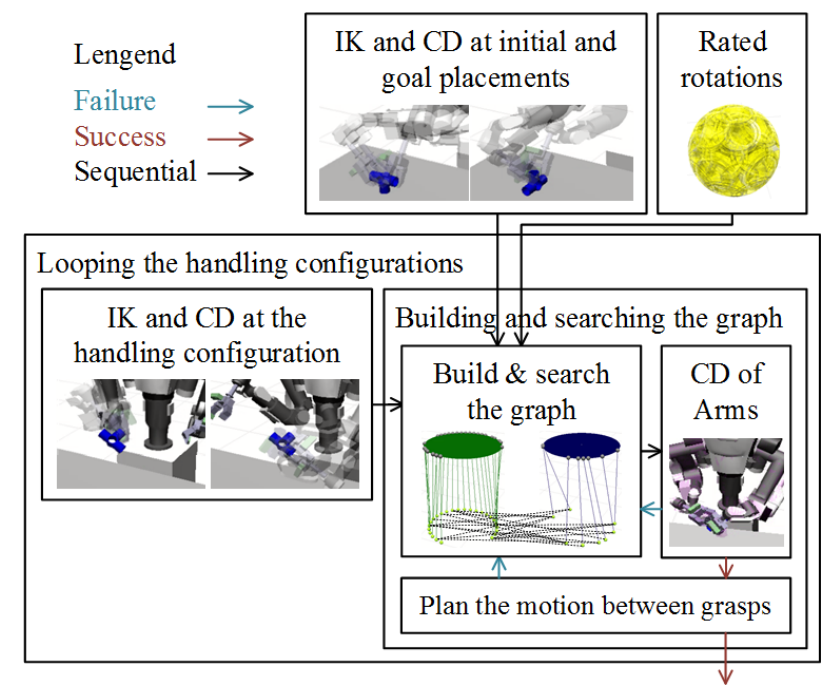

Fig. 7. Searching the dual-arm regrasp graph. In the "looping the handling configurations" box, we loop the handling configurations and build the regrasp graph. The graph is rebuilt for each handling configuration. In the "building and searching the graph" box, we use the same searching method shown in Fig 6 Checking the IK and collision at initial and goal placements and rating the the rotation of handling configurations are performed before starting the loop.

\section{EXPERIMENTS AND ANALYSIS}

We analyze the performance of our algorithms using simulation. The model of our objects are based on an "L"- shape block, a "box"-shape part, and a "T"-shape tube (see Fig 10, Our robot platform is the HiroNX 1 and our simulation software is based on Choreonoid and its graspPlugin ${ }^{2}$ Real-world executions of single-arm regrasp using KINECT were published in our previous work ${ }^{3}$ This paper concentrates on simulation and the comparison of single-arm and dual-arm regrasp. It doesn't present the details of those implementations.

\section{A. Respective analysis}

Fig 8 shows the snapshots of single-arm and dual-arm regrasp. The initial configuration of these results is an object placing on a table, and the goal is the object placing at the same place with a different placement. The robot is required to reorientate the object with either single-arm or dual-arm regrasp. We draw the key frames of the transit and transfer motions in the upper row of each sub-figure, and draw the correspondent regrasp graph in the lower row. The correspondence between the nodes, edges, and the key frame figures are marked with red circles and segments, and are written on the upper-left corner of each sub-figure in the lower row (See the caption for details). The results of other objects are shown in the video attachment.

Fig 9 shows the time cost of graph search and motion planning in single-arm and dual-arm regrasp. Each column of the table is the result of one regrasp task where the initial and goal placements are drawn in the top. The figure is divided into two parts by a dash line where the upper part is singlearm regrasp. The time cost of singl-arm graph search and its number of research times are shown in the first data row. The time cost of motion planning are shown in the other data rows. Graph search takes less than $10 s$ and an average of 6 times of re-search for this object. Motion planning is less than $1 s$ for both transit and transfer motion. The results of simple pick-and-place task where no reorientation is required are shown in the last column. It takes $2 s$ for graph search and less than $0.1 s$ for motion planning. The lower part of Fig 9 is the cost of dual-arm regrasp. The first row shows the time cost of IK solving and collision detection at initial and goal placements. The second row shows the number of rotation tried during searching. The first two rows correspond to the top two boxes in Fig 7. The third row shows the time cost of searching the master sub-graph, and the fourth row shows the time cost of searching the slave sub-graph. Depending on the number of intermediate configurations tried, the number of data in the third row changes. The rows 5-8 are the time cost of motion planning. The most time-consuming part is the IK solving and collision detection at initial and goal placements. It is $10.02 s$ in the worst case. The time cost is well acceptable consdiering that we sample the object surface using $0.01 \mathrm{~m}$ granularity and sample the approaching directions at every $\frac{\pi}{4}$ radian. The second time-consuming part is searching the master graph. Since we build the graph for each handling configuration, we might test all possible rotations which costs

\footnotetext{
${ }^{1}$ See http://nextage.kawada.jp/en/

${ }^{2}$ See http://choreonoid.org/en//http://choreonoid.org/GraspPlugin/i/?q=en

${ }^{3}$ See a video at http://youtu.be/Mlgl8BmvfPc
} 


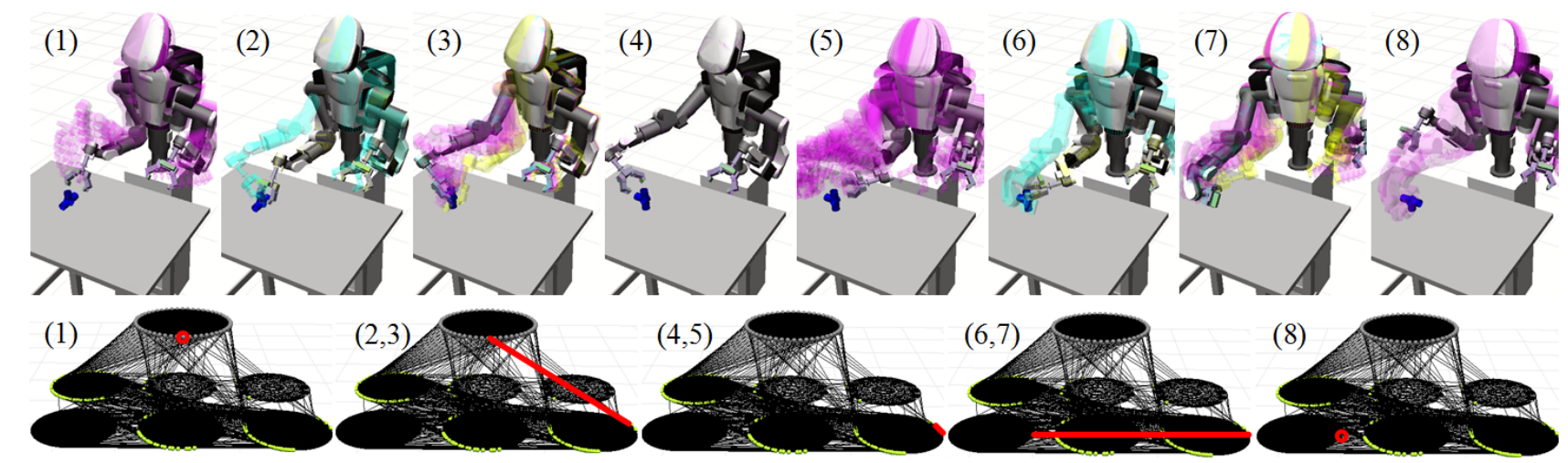

(a) The snapshots of single-arm regrasp. In (1), the robot does motion planning to grasp the object. The virtual robot sequences drawn in purple are the key frames of the planned motion. It belongs to the transit motion type. On the regrasp graph (see the second row), it is a single node in one circle (marked with red color). In (2), the robot finds the initial and goal of a transfer motion. The virtual robot drawn in yellow color shows the initial and the virtual robot drawn in cyan color shows the goal. The robot does motion planning to perform this transfer motion in (3). On the regrasp graph, (2) and (3) correspond to an edge connecting two circles. In (4) and (5), the robot "regrasps" the object by changing the grasps. The motion in these figures is transit. They correspond to an edge in the same circle on the regrasp graph. (6) and (7) is the same as (2) and (3), and they are the task and motion planning result of the second transfer motion. They correspond to an edge connecting two circles. In (8), the robot does the final motion planning to retract the hand to standard pose. It is a single node on the regrasp graph.

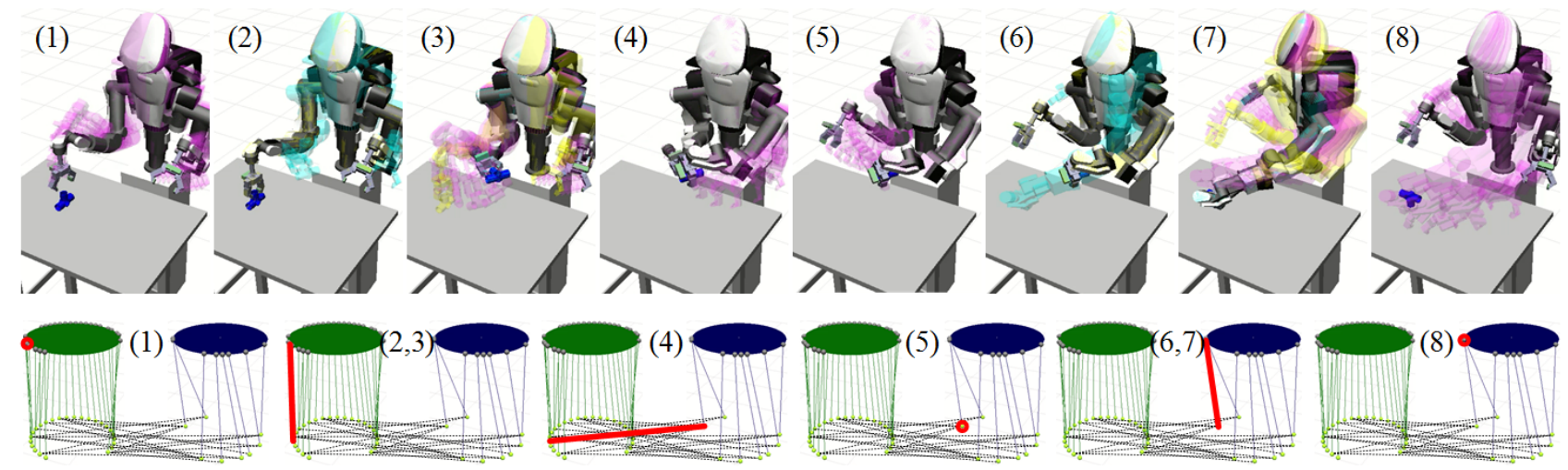

(b) The snapshots of dual-arm regrasp. In (1), the robot does transit motion planning to grasp the object with master hand. It corresponds to a node in the upper circle (see the lower row of figures). In (2) and (3), the robot transfers the object into a handling configuration with master hand. It corresponds to an edge connecting the upper and lower layers in the master sub-graph. In (4), the robot "regrasp" the object with the slave hand. The motion is transit and corresponds to an edge connecting the lower circles of the two sub-graphs. In (5), the robot retract the master hand. In (6) and (7), the robot transfers the object into the goal placement on the table with the slave hand. It corresponds to an edge connecting the lower and upper layers in the slave sub-graph. Finally, the robot transit its slave hand back in (8). Like (a), when the robot does motion planning, we draw a sequence of virtual robots in purple color. When the robot does transfer motion, we draw the initial and goal virtual robots in yellow and cyan colors.

Fig. 8. Snapshots of the robot regrasping the "T"-shape tube using single-arm and dual-arm regrasp in simulation.

as much as 6 minutes in the worst case. This estimation is based on 352 rotations and $1 s$ time cost per search. During the experiments, none of the search exceeds 3minutes. The algorithms are fast enough to deal with large number of grasps and exploded combinatorics and can be run thousands of times for comparison.

\section{B. Comparison}

In order to compare single-arm and dual-arm regrasp, we set the initial and goal of the object at a fixed position and use a random rotation around the normal of the table surface to generate initial and goal placements. The robot is required to reorientate the object from the initial placement to the goal using single-arm or dual-arm regrasp.

Fig 10 shows the results of the comparison. The left column of each table lists the initial placements and the upper row lists the goal placements. The positions are fixed but we set a random yaw angle to the initial placement to increase uncertainty. At each grid, the blue data shows the successful rate and time cost of single-arm regrasp. In constrast, the red data shows those of the dual-arm regrasp. Like our expectation, dual-arm regrasp has good performance in most cases (see the area shaded in green color in Fig 10. It is sometimes a bit slow, but the extra time cost is acceptable and the successful rate is much higher than single-arm regrasp. In a few cases, however, dual-arm regrasp has bad performance (see the area shaded in purple color). This usually happens to the simple pick-and-place tasks where no reorientation is needed: See (row 1, column 1) and (row 4, column 4) of the "L"-shape tube, (row 1, column 1) of the "box"-shape part, and (row 2, column 2) of the "T"-shape tube. In these cases, the grasps of the master hand and the slave hand are on the upper part of the object to ensure it can be placed down to the same placement. They overlap with each other 


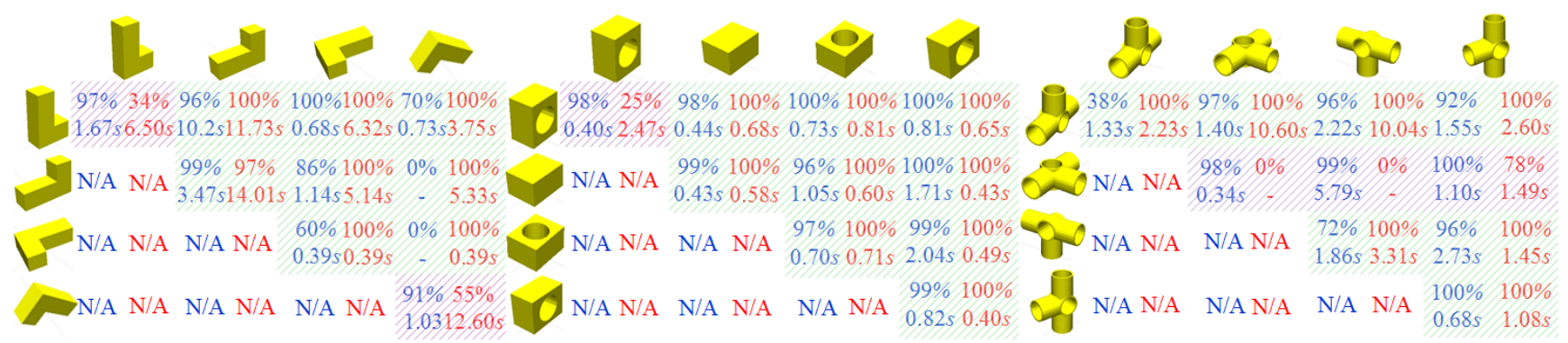

Fig. 10. Average successful rates and costs of single-arm and dual-arm regrasp. Dual-arm regrasp has higher performance at the tasks under the green shade, and has lower perfomrance at the tasks under the purple shade.

\begin{tabular}{lcccc} 
& & 200 & 0 \\
Graph Search & $5.32 s / 11$ & $2.57 s / 6$ & $2.41 s / 6$ & $1.51 s / 1$ \\
Initial Transit & $0.11 s$ & $0.05 s$ & $0.17 s$ & $0.07 s$ \\
Transfer1 & $0.36 s$ & $0.12 s$ & $0.80 s$ & $0.08 s$ \\
Transit1 & $0.09 s$ & $0.23 s$ & - & - \\
Transfer2 & $0.36 s$ & $0.50 s$ & - & - \\
End Transit & $0.34 \mathrm{~s}$ & $0.13 \mathrm{~s}$ & $0.50 \mathrm{~s}$ & $0.07 s$ \\
\hline IK/CD at init and goal & $4.92 s$ & $9.46 s$ & $8.03 s$ & $10.02 s$ \\
\# of Intermediate confs & 1 & 6 & 1 & 3 \\
Master sub-graph search & $0.02 s$ & $1.32 s$ & $0.01 s$ & - \\
\&Intermediate checking & & $5.24 s$ & & $0.15 s$ \\
& & $0.75 s$ & & \\
& & $0.03 s$ & & \\
Slave sub-graph search & $0.16 s$ & $0.02 s$ & $0.19 s$ & $0.19 s$ \\
Initial Transit & $0.13 s$ & $0.09 s$ & $0.24 s$ & $0.09 s$ \\
Transfer1 & $0.36 s$ & $0.46 s$ & $0.62 s$ & $0.37 s$ \\
Transfer2 & $0.42 s$ & $0.46 s$ & $0.90 s$ & $0.38 s$ \\
End Transit & $0.26 s$ & $0.10 s$ & $0.18 s$ & $0.98 s$ \\
& & & &
\end{tabular}

Fig. 9. Time cost of graph search and motion planning in single-arm (above the dashline) and dual-arm regrasp (below the dash line).

and collide during handling. (row 2, column 3) and (row 2, column 4) of the tube are also difficult to dual-arm regrasp. The reason is similar - the grasps of the master hand at the initial placement overlap with the grasps of the slave hand at the goal placement, and the master hand and the slave hand collide during handling. The results demonstrate that dual-arm regrasp is not necessarily better than single-arm regrasp. The performance depends on object shapes and the overlapping of grasps.

\section{Conclusions ANd Future Work}

In this paper, we develop efficient algorithms for singlearm and dual-arm regrasp and run the algorithms thousands of times to compare their performance. We confirm the algorithms are fast to deal with large number of grasps and find dual-arm regrasp is not necessarily better than singlearm regrasp: It depends on object shapes and the overlapping of grasps. We expect the results will help practitioners to choose proper number of arms.

In the future, we would further explore the features to choose the arm numbers using deep learning and develop algorithms to predict whether a robot should use a single arm or dual arms. 